\title{
Credible measures of resolution limits
}

\author{
Zbyszek Otwinowski ${ }^{1}$, Dominika Borek ${ }^{1}$, Marcin Cymborowski², Przemek Porebski², Wladek \\ Minor $^{2}$ \\ ${ }^{1}$ Department of Biophysics, University of Texas Southwestern Medical Center, Dallas, TX, 75390; \\ Zbyszek.Otwinowski@UTSouthwestern.edu \\ ${ }^{2}$ Molecular Physiology and Biological Physics, University of Virginia, Charlottesville, VA 22908, \\ wladek@iwonka.med.virginia.edu
}

In structural biology, the resolution limit is broadly used as a measure of how detailed the experimental information is, or more formally, defines the radius (inverse of resolution) of useful information in reciprocal space. This measure is conceptually independent from data quality, described for instance by the signal-to-noise ratio (SNR), but in practice we use cutoffs based on data quality to define a resolution limit that is usually expressed as a single number.

However, there are several problems in how a resolution limit is calculated and used as an indicator:

[1] Data quality can be anisotropic because direction can affect crystal lattice (dis)order and because experimental factors have directional dependence on the amount and quality of data. Such situations are quite frequent in both crystallography and cryo-EM and reporting practices are still evolving.

[2] The standards defining data quality cutoffs are not consistent within and between the fields of X-ray crystallography and cryo-EM. All cutoffs either implicitly or explicitly rely upon SNR considerations, but these can be applied either to structure factors or structure factor amplitudes squared. This duality has complex consequences because the use of structure factors or structure factor amplitudes squared is not equivalent at low SNR.

[3] Because resolution has high significance in reporting, it can be subject to cherry picking from multiple alternative analyses of the data. In crystallography, the problem is less pronounced because the experimental dataset and its limit are assessed prior to refinement and alternative datasets are assessed mainly by refinement quality. In cryo-EM, the problem is much more severe because the resolution limit is established from internal measures of $3 D$ reconstruction statistics where there are many more possibilities, with large, pseudo-random fluctuations in resolution limit estimators.

I will discuss new approaches for defining resolution in general and in particular for anisotropic data. 